\title{
A 20-year audit of retinoblastoma treatment outcomes
}

\author{
Ahmet Kaan Gündüz $z^{1}$ Ibadulla Mirzayev $\mathbb{D}^{1} \cdot$ Emine Temel $\mathbb{D}^{1} \cdot$ Emel Ünal $^{2} \cdot$ Nurdan Taçyıldız $^{2}$ • \\ Handan Dinçaslan ${ }^{2} \cdot$ Serdal Kenan Köse ${ }^{3} \cdot$ Funda Seher Özalp Ateş ${ }^{3} \cdot$ Mehmed Uğur Işık (1) 4
}

Received: 8 August 2019 / Revised: 10 April 2020 / Accepted: 15 April 2020 / Published online: 6 May 2020

(c) The Author(s), under exclusive licence to The Royal College of Ophthalmologists 2020

\begin{abstract}
Objectives To evaluate the long-term treatment outcomes in intraocular retinoblastoma (RB) including the associated factors for eventual treatment with external beam radiotherapy (EBRT) and enucleation as well as to analyse the risk factors for metastasis and death in extraocular RB.

Methods Retrospective analysis of 390 eyes from 256 (89.8\%) intraocular RB and 29 (10.2\%) extraocular RB cases diagnosed and treated between October 1998 and May 2018 at one of the largest tertiary care centers in Turkey.

Results Of 351 intraocular RB eyes, 53.3\% had group D/E disease at presentation. 75 (21.4\%) of 351 eyes underwent primary enucleation. Of the remaining 276 eyes undergoing eye-conserving treatments, 201 (72.8\%) were salvaged. Most of these eyes were treated using intravenous chemotherapy and/or focal treatments [transpupillary thermotherapy (TTT) and cryotherapy] initially. EBRT was eventually required in $48(17.4 \%)$ eyes and secondary enucleation in $75(27.2 \%)$ eyes. At mean follow-ups of 76.7 and 39.7 months for intraocular and extraocular RB cohorts, respectively, 180 (46.2\%) eyes underwent primary/secondary enucleation and exenteration. Overall, 13 cases developed metastasis and 9 died. Two patients with trilateral RB also expired. Multivariable risk factors for enucleation were the presence of vitreous seeds $(p<0.001)$, absence of EBRT administration $(p=0.033), 5-9$ TTT applications compared with no TTT $(p=0.031)$, and each $1 \mathrm{~mm}$ increase in tumour base diameter $(p<0.001)$. Univariate factors predictive of metastasis were the presence of extraocular RB detected by imaging methods $(p<0.001)$ and extrascleral/optic nerve cut end involvement at histopathological examination $(p<0.001)$. Conclusions In our series, $72.8 \%$ of the intraocular RB eyes undergoing eye-conserving treatments were saved. The globe salvage rate for all intraocular and extraocular RB eyes was $53.8 \%$ and the overall survival rate was $96.1 \%$.
\end{abstract}

\section{Introduction}

Retinoblastoma (RB) is the most common intraocular malignant tumour in the paediatric age group [1]. It accounts for $\sim 4 \%$ of all paediatric malignancies [2]. RB

Supplementary information The online version of this article (https:// doi.org/10.1038/s41433-020-0898-9) contains supplementary material, which is available to authorized users.

Ahmet Kaan Gündüz

drkaangunduz@gmail.com

1 Department of Ophthalmology, Ankara University Faculty of Medicine, Ankara, Turkey

2 Departmant of Pediatrics, Ankara University Faculty of Medicine, Ankara, Turkey

3 Department of Biostatistics, Ankara University Faculty of Medicine, Ankara, Turkey

4 Şanlıurfa Balıklıgöl State Hospital, Şanlıurfa, Turkey carries a significant risk for mortality. Worldwide, RB survival is $\sim 30 \%$ in Africa, $60 \%$ in Asia, $80 \%$ in Latin America, 95\% in Europe, and 97\% in North America [1]. Delayed diagnosis and treatment result in advanced intraocular RB and increase the risk of extraocular spread [3]. During the past 30 years, conceptual changes took place in the management of RB. There has been a shift from globe-sacrificing methods and external beam radiotherapy (EBRT) to globe-saving alternatives employing chemotherapy.

The aims of this study were to evaluate the risk factors leading to treatment with EBRT, enucleation, metastasis, and death in patients with intraocular RB and to analyse the outcomes after treatment in patients with extraocular RB.

\section{Materials and methods}

All cases diagnosed with $\mathrm{RB}$ and received treatment and follow-up on the Ocular Oncology Service, Department of 
Ophthalmology, Ankara University Faculty of Medicine, Ankara, Turkey between October 1998 and May 2018 were retrospectively evaluated. Cases who had their initial treatment at other centers and referred to us for further management were excluded from this study. Risk factors for EBRT, enucleation, metastasis, and death were analysed. The study was performed according to the tenets of the Declaration of Helsinki and informed consent was obtained from all cases.

\section{Intraocular retinoblastoma}

Patients with intraocular RB underwent examination under general anaesthesia by the senior author (AKG) at baseline and at every month during treatment process until tumour regression was noted. The frequency of follow-up visits was subsequently adjusted according to patient needs. All cases were classified according to International Classification of RB (ICRB) (Supplemental Table 1) [4]. Brain magnetic resonance imaging (MRI) studies were routinely performed at baseline, twice yearly up to 5 years of age and then once yearly to screen for pinealoblastoma or trilateral RB. Lumbar puncture and bone marrow aspiration studies were performed as deemed necessary by the paediatric oncologists.

Bilateral RB cases (groups B-E) underwent intravenous chemotherapy (CRD) consisting of vincristine, etoposide, and carboplatin administered as six cycles at 4 week intervals (Supplemental Table 2). Transpupillary thermotherapy (TTT) and cryotherapy were used after the second or third CRD cycle to the regressing tumours. Group A and some group B RBs underwent TTT and cryotherapy only.

Retinal tumours, vitreous or subretinal seeds demonstrating no significant regression after the second or third CRD cycle were considered as unresponsive disease. Eyes with unresponsive disease underwent Ruthenium-106 (Ru-106) plaque radiotherapy, EBRT, secondary enucleation, intraarterial chemotherapy (IAC), and/or intravitreal chemotherapy (IVC). For EBRT, a total dose of 30-40 Gy was used. For Ru-106 plaque radiotherapy, an apex dose of 40 Gy was prescribed. The chemotherapeutic agents used in IAC included melphalan (3-7.5 mg), topotecan ( $0.5-2 \mathrm{mg}$ ) and carboplatin (20-30 mg). The infusion was administered over 30 minutes. IAC was given for 1-3 cycles on a monthly basis. The chemotherapeutic agents used in the IVC included melfalan $(20-30 \mu \mathrm{g} / 0,05 \mathrm{ml})$ and topotecan $(20 \mu \mathrm{g} / 0,05 \mathrm{ml})$. IVC was usually administered as biweekly or monthly cycles for 2-3 times. Eyes that occasionally demonstrated satisfactory regression after the first IVC cycle did not receive any further IVC treatment. All chemotherapy drugs used had approval by the Pediatric Oncology Review Board.

Regrowth of retinal tumours, vitreous or subretinal seeds after initial response was defined as recurrence. New tumour was defined as a tumour that was not contiguous with the pre-existing tumours and not noted at baseline examination. Recurrent and new tumours were treated by $2-4$ additional CRD cycles, TTT, cryotherapy, Ru-106 plaque radiotherapy, EBRT, IAC, and/or IVC.

Unilateral RB (groups D-E) underwent CRD/enucleation in the earlier phases of our study and IAC during the later phases. Groups B-C eyes received CRD in the earlier phases of the study and IAC in the later phases. Group A and some group B eyes received TTT and cryotherapy only. Treatment for nonresponding, recurrent and new tumours were the same as for bilateral tumours with the exception of avoidance of EBRT in unilateral cases. Despite these medical preferences, the decision for final treatment was made based on extensive discussion with the patient's family.

For eyes that underwent enucleation, high-risk histopathologic features included anterior chamber invasion, choroidal infiltration $>3 \mathrm{~mm}$, scleral infiltration, retrolaminar optic nerve invasion, and any degree of combined choroidal and optic nerve invasion (laminar or retrolaminar). High-risk eyes received adjuvant treatment consisting of 4 cycles of vincristine, etoposide, and carboplatin chemotherapy.

\section{Extraocular and metastatic retinoblastoma}

According to the International Retinoblastoma Staging System (IRSS), patients with extraocular RB had extraocular extension on pathologic examination (extrascleral/cut end of optic nerve involvement) after enucleation (Stage II) or demonstrated clinically evident extraocular extension on MRI at presentation (Stage III) [3]. These cases received incisional biopsy of the orbital mass if the diagnosis was not certain, intensive systemic chemotherapy, and orbital EBRT followed by enucleation/exenteration if necessary (Supplemental Table 3). Autologous stem cell rescue (ASCR) was applied in necessary cases.

Brain MRI, bone marrow aspiration, lumbar puncture, and other required imaging studies were concurrently performed to evaluate the presence of metastases in these cases. If there was evidence of systemic metastasis to the central nervous system (CNS) and/or other sites, intensive chemotherapy, EBRT to the relevant sites, and ASCR were administered (Supplemental Table 3). Trilateral RB cases were treated similar to those with CNS metastasis.

\section{Statistical analysis}

All statistical analyses were performed using SPSS for Windows 11.5 (SPSS Inc, Chicago, IL, USA). Kolmogorov-Smirnov test was used to assess the assumption of normality. The continuous variables that do not have 
normal distribution were expressed as mean (median, range). Categorical variables were summarized as counts (percentages).

Univariate logistic regression analysis was used to analyse the factors leading to treatment with EBRT, enucleation, and metastasis, whereas univariate Cox proportional hazard model was used for death. A two-sided $p$ value < 0.05 was considered as statistically significant.

Multivariable logistic regression model was used to predict potential risk factors of EBRT and enucleation. The variables which had a significance level of $p<0.20$ from the univariate analysis were identified as candidate variables for the multivariable model. Multivariable logistic regression model was created with Backward LR method. Kaplan-Meier survival analysis and logrank test were used for assessing the survival rates in patients with intraocular RB who underwent EBRT and enucleation versus who did not undergo these treatments. Kaplan-Meier analysis were also used to predict the survival in extraocular RB patients.

\section{Results}

During the study period from October 1998 to May 2018, 285 cases with 390 eyes affected with RB were enrolled in this study. In total, $158(55.4 \%)$ patients were male and 127 $(44.6 \%)$ were female. The mean age was 21.8 (median:12, range:1-204) months. In total, 26 of $285(9.1 \%)$ cases had a family history of RB. RB was unilateral in $180(63.2 \%)$ cases and bilateral in 105 (36.8\%). Of 285 cases, 256 $(89.8 \%)$ had intraocular RB and 29 (10.2\%) had extraocular RB.

\section{Intraocular retinoblastoma}

Patient demographics and clinical staging in eyes with intraocular RB are given in Table 1. Of 256 cases with intraocular RB, 161 (62.9\%) had unilateral disease and 95 $(37.1 \%)$ had bilateral disease. The mean largest tumour base diameter was 13.4 (median:10, range: 1.0-35) $\mathrm{mm}$ and the mean tumour thickness was 4.8 (median:4, range: 1.0-15) $\mathrm{mm}$.

Table 2 summarizes the treatment methods used in intraocular RB. Seventy-five $(21.4 \%)$ eyes underwent primary enucleation. The remaining 276 eyes had eyeconserving treatments, including 254 with CRD, 19 with TTT and cryotherapy only, and 3 with IAC only. The mean number of CRD administrations was 6.2 (median: 6; range: 2-12). TTT was used as supplemental treatment in 141 $(55.5 \%)$ eyes and cryotherapy in $128(50.4 \%)$ eyes. The mean number of TTT and cryotherapy applications were 2.8 (median: 2, range: 1-9) and 3.2 (median: 2, range: 1-15), respectively.
Table 1 Patient demographics and clinical staging in eyes with intraocular retinoblastoma.

\begin{tabular}{ll}
\hline Mean age (range) & $21.4(1-204)$ \\
Sex (male/female) & $142 / 114$ \\
Laterality (unilateral/bilateral) & $161 / 95$ \\
ICRB group & Eyes $(\%)$ \\
A & $22(6.3)$ \\
B & $114(32.5)$ \\
C & $28(8.0)$ \\
D & $90(25.6)$ \\
E & $97(27.6)$ \\
\hline
\end{tabular}

ICRB international classification of retinoblastoma.

Table 2 Treatment methods used in intraocular RB

\begin{tabular}{ll}
\hline Treatment methods & $\begin{array}{l}\text { Number of eyes receiving treatment/ } \\
\text { Total number of eyes in cohort }(\%)\end{array}$ \\
\hline CRD & $254 / 276(92.0)$ \\
TTT & $156 / 276(56.5)$ \\
Cryotherapy & $138 / 276(50.0)$ \\
Primary enucleation & $75 / 351(21.4)$ \\
$\quad$ Unilateral RB & $64 / 161(39.8)$ \\
$\quad$ Bilateral RB & $11 / 190(5.8)$ \\
Secondary enucleation & $75 / 276(27.2)$ \\
$\quad$ Unilateral RB & $37 / 97(38.1)$ \\
$\quad$ Bilateral RB & $38 / 179(21.2)$ \\
EBRT & $48 / 276(17.4)$ \\
IAC & $25 / 276(9.1)$ \\
$\quad$ Primary & $3 / 276(1.1)$ \\
$\quad$ Secondary & $22 / 276(8.0)$ \\
Focal treatments only (TTT, & $19 / 276(6.9)$ \\
cryotherapy) & \\
Ruthenium-106 plaque & $18 / 276(6.5)$ \\
radiotherapy & \\
IVC & $11 / 276(4.0)$
\end{tabular}

$C R D$ chemoreduction, $T T T$ transpupillary thermotherapy, $R B$ retinoblastoma, EBRT external beam radiotherapy, IAC intraarterial chemotherapy, IVC intravitreal chemotherapy.

Recurrent tumours were observed in $71(25.7 \%)$ eyes. The mean recurrent tumour number was 2.5 (median: 1, range: $1-4)$. The mean time elapsed to the development of recurrent tumours was 11.4 (median: 8, range: 2-69) months. $22(31.0 \%)$ of 71 eyes had second recurrence, 11 $(50.0 \%)$ of 22 eyes had third recurrence and $3(27.3 \%)$ of 11 eyes had fourth recurrence. New tumours were noted in 59 $(21.4 \%)$ eyes. The mean new tumour number was 1.2 (median: 1, range: 1-3). The mean time recorded for the development of new tumours was 9.7 (median: 6; range: 1-61) months. 
The mean follow-up was 76.7 (median: 66, range: 1-268) months. Of 276 eyes undergoing CRD, focal treatments or IAC, $201(72.8 \%)$ could be saved. In the subgroup of 19 eyes undergoing only focal treatments without CRD, all eyes were salvaged. After eye-conserving treatments, additional measures for unresponsive, recurrent and new tumours included secondary enucleation [75 eyes $(27.2 \%)$ (37 unilateral, 38 bilateral RB)], EBRT [48 eyes (17.4\%)], IAC [22 eyes (8.0\%)], Ru-106 plaque radiotherapy [18 eyes $(6.5 \%)]$, and IVC [11 eyes (4.0\%)] (Table 2). The globe salvage rate was $68.8 \%$ (33 of 48 eyes), $45.5 \%$ (10 of 22 eyes), $50.0 \%$ ( 9 of 18 eyes), $72.7 \%$ ( 8 of 11 eyes) in eyes which underwent EBRT, IAC, Ru-106 plaque radiotherapy, and IVC as end point (secondary) treatment respectively.

One of the three eyes undergoing primary IAC had group C, 1 had group D, and 1 had group E RB. Of the 22 eyes which received secondary IAC, 4 eyes had group B, 3 eyes had group C, 11 eyes had group D, and 4 eyes had group E RB. Among 25 eyes undergoing primary or secondary IAC, 12 eyes received 3 cycles, 4 eyes received 2 cycles, and 9 eyes received 1 cycle. All eyes which underwent primary IAC were salvaged. After secondary IAC, the globe salvage rate was $50.0 \%$ in group B (2 of 4 eyes), $66.7 \%$ in group C ( 2 of 3 eyes), $45.5 \%$ in group D (5 of 11 eyes), and $25.0 \%$ in group $\mathrm{E}$ ( 1 of 4 eyes).

Of the total 11 eyes which received IVC, 2 eyes had group B RB at initial presentation but progressed to group C at most advanced stage. The remaining 1 eye had group C, 4 eyes had group D, and 4 eyes had group E RB. In total, 3 of 11 eyes received 4 IVC cycles, 1 eye received 3 cycles, 4 eyes received 2 cycles, and 3 eyes received 1 cycle. The globe salvage rate was $100 \%$ in groups B and C, $75.0 \%$ in group D (3 of 4 eyes), and 50.0\% in group E (2 of 4 eyes) after IVC.

The results (odds ratio (OR), 95\% confidence interval (95\% CI), and $p$ value) of univariate and multivariable logistic regression analysis for eventual treatment with EBRT are shown in Table 3. Kaplan-Meier curves show that there was no statistically significant difference in survival between patients who had EBRT versus those who did not $(p=0.575)$ (Fig. 1a).

The globe salvage rate was $100 \%$ in group A (22 of 22 eyes), $86.0 \%$ in group B (98 of 114 eyes), $85.7 \%$ in group C (24 of 28 eyes), $45.6 \%$ in group D (41 of 90 eyes), and $16.5 \%$ in group $\mathrm{E}$ (16 of 97 eyes). Of 75 primary enucleations, $8(10.7 \%)$ eyes demonstrated high-risk histopathologic features. Of 75 secondary enucleations, 10 (13.3\%) eyes demonstrated high-risk histopathologic features. These cases were treated with 4 additional doses of adjuvant chemotherapy. In total, 49 of 180 (27.2\%) bilateral $\mathrm{RB}$ eyes were enucleated versus 101 of 161 (62.7\%) unilateral $\mathrm{RB}$ eyes.

The results (OR, 95\% CI, and $p$ value) of univariate and multivariable logistic regression analysis for treatment with enucleation are shown in Table 4. Kaplan-Meier curves show that there was no statistically significant difference in survival between patients who underwent enucleation versus those who did not $(p=0.760)$ (Fig. 1b).

One patient with intraocular RB and retrolaminar optic nerve invasion found at histopathological examination developed CNS metastasis and eventually expired. One patient with trilateral $\mathrm{RB}$ in the intraocular group also died. There was no other death recorded in the intraocular $\mathrm{RB}$ group and the survival rate was $99.2 \%$. Secondary

Table 3 Univariate and multivariable logistic regression analysis for eventual treatment with EBRT.

\begin{tabular}{|c|c|c|c|c|c|c|}
\hline \multirow[t]{2}{*}{ Risk factors for EBRT } & \multicolumn{3}{|c|}{ Univariate logistic regression analysis } & \multicolumn{3}{|c|}{ Multivariable logistic regression analysis } \\
\hline & Crude OR & $95 \% \mathrm{CI}$ & $p$ value & Adjusted OR & $95 \% \mathrm{CI}$ & $p$ value \\
\hline Age (Each 1 year increase) & 1.010 & $1.000-1.019$ & 0.052 & 1.021 & $1.009-1.032$ & $<0.001$ \\
\hline Tumour group by ICRB (A) & & & 0.029 & - & - & 0.029 \\
\hline B & 2.432 & $0.530-11.148$ & 0.253 & 2.752 & $0.499-15.165$ & 0.245 \\
\hline $\mathrm{C}$ & 4.725 & $0.907-24.603$ & 0.065 & 9.023 & $1.401-58.101$ & 0.021 \\
\hline $\mathrm{D}$ & 2.660 & $0.573-12.349$ & 0.212 & 6.709 & $1.138-39.562$ & 0.036 \\
\hline $\mathrm{E}$ & 0.933 & $0.185-4.719$ & 0.934 & 3.676 & $0.537-25.180$ & 0.185 \\
\hline Presence of subretinal seeds & 0.585 & $0.333-1.025$ & 0.061 & - & - & - \\
\hline Plaque radiotherapy & 2.754 & $0.993-7.637$ & 0.052 & 3.331 & $1.067-10.396$ & 0.038 \\
\hline Absence of enucleation & 0.504 & $0.284-0.893$ & 0.019 & 0.427 & $0.198-0.921$ & 0.030 \\
\hline $\begin{array}{l}\text { Number of TTT } \\
\text { applications }(=0)\end{array}$ & & & 0.014 & & & 0.014 \\
\hline $1-4$ & 2.133 & $1.194-3.809$ & 0.011 & 2.149 & $1.095-4.218$ & 0.026 \\
\hline $5-9$ & 2.689 & $1.043-6.932$ & 0.041 & 4.188 & $1.394-12.580$ & 0.011 \\
\hline
\end{tabular}

$E B R T$ external beam radiotherapy, $O R$ odds ratio, $C I$ confidence interval, ICRB international classification of retinoblastoma, $T T T$ transpupillary thermotherapy. 


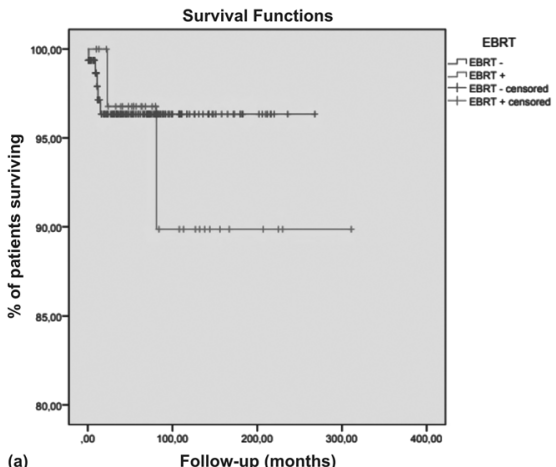

Fig. 1 Kaplan-Meier survival curves for external beam radiotherapy (EBRT), enucleation, and extraocular extension. a Kaplan-Meier curves show the survival rates in patients undergoing EBRT versus those who did not have EBRT. Survival was $100 \%$ at 1 year, $96.8 \%$ at 5 years, $89.9 \%$ at 10 years in patients who had EBRT. Survival was $97.1 \%$ at 1 year, $96.3 \%$ at 5 years, $96.3 \%$ at 10 years in patients who did not undergo EBRT. b Kaplan-Meier curves show the survival rates in patients who underwent enucleation versus those who
Survival Functions

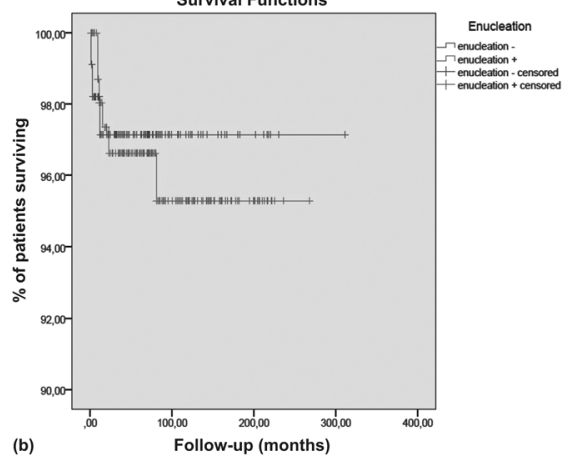

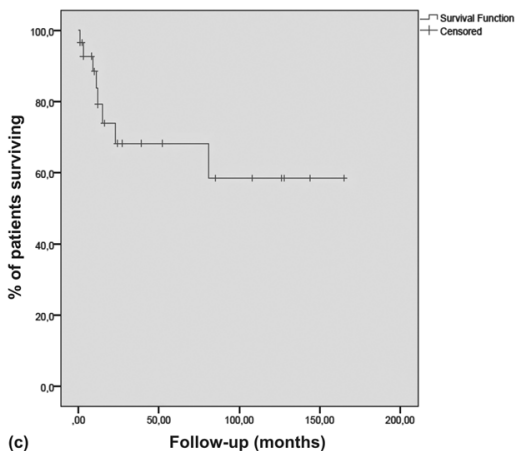

did not undergo enucleation. Survival was $98.0 \%$ at 1 year, $96.6 \%$ at 5 years, $95.3 \%$ at 10 years in patients who underwent enucleation. Survival was $97.1 \%$ at 1 year, $97.1 \%$ at 5 years, $97.1 \%$ at 10 years in patients who did not undergo enucleation. c Kaplan-Meier curve shows the survival rates in patients with extraocular retinoblastoma. Survival was $79.2 \%$ at 1 year, $68.2 \%$ at 5 years, and $58.5 \%$ at 10 years after initial diagnosis.

Table 4 Univariate and multivariable logistic regression analysis for treatment with enucleation.

\begin{tabular}{|c|c|c|c|c|c|c|}
\hline \multirow[t]{2}{*}{ Risk factors for enucleation } & \multicolumn{3}{|c|}{ Univariate logistic regression analysis } & \multicolumn{3}{|c|}{$\begin{array}{l}\text { Multivariable logistic regression } \\
\text { analysis }\end{array}$} \\
\hline & Crude OR & $95 \% \mathrm{CI}$ & $p$ value & Adjusted OR & $95 \% \mathrm{CI}$ & $p$ value \\
\hline Age & 1.007 & $0.998-1.016$ & 0.113 & - & - & - \\
\hline Family history & 0.292 & $0.136-0.629$ & 0.002 & - & - & - \\
\hline Tumour class by ICRB (A) & & & $<0.001$ & - & - & - \\
\hline $\mathrm{B}$ & 3.485 & $0.439-27.681$ & 0.238 & - & - & - \\
\hline $\mathrm{C}$ & 4.583 & $0.496-42.353$ & 0.180 & - & - & - \\
\hline $\mathrm{D}$ & 23.956 & $3.101-185.057$ & 0.002 & - & - & - \\
\hline $\mathrm{E}$ & 112.750 & $14.165-897.437$ & $<0.001$ & - & - & - \\
\hline Presence of vitreous seeds & 9.510 & $5.917-15.283$ & $<0.001$ & 4.246 & $2.260-7.976$ & $<0.001$ \\
\hline Presence of subretinal seeds & 4.709 & $3.064-7.235$ & $<0.001$ & - & - & - \\
\hline Absence of EBRT & 0.504 & $0.284-0.893$ & 0.019 & 0.411 & $0.182-0.931$ & 0.033 \\
\hline $\begin{array}{l}\text { Number of cryotherapy } \\
\text { applications }(=0)\end{array}$ & & & $<0.001$ & - & - & - \\
\hline $1-4$ & 0.170 & $0.096-0.299$ & $<0.001$ & - & - & - \\
\hline $5-9$ & 0.587 & $0.239-1.440$ & 0.245 & - & - & - \\
\hline $10-15$ & 0.782 & $0.155-3.948$ & 0.766 & - & - & - \\
\hline $\begin{array}{l}\text { Number of TTT } \\
\text { applications }(=0)\end{array}$ & & & $<0.001$ & - & - & 0.028 \\
\hline $1-4$ & 0.276 & $0.169-0.450$ & $<0.001$ & 0.719 & $0.363-1.424$ & 0.344 \\
\hline $5-9$ & 0.600 & $0.265-1.357$ & 0.220 & 3.121 & $1.113-8.751$ & 0.031 \\
\hline $\begin{array}{l}\text { Tumour base diameter }(\mathrm{mm}) \\
\text { (Each } 1 \mathrm{~mm} \text { increase) }\end{array}$ & 1.202 & $1.142-1.264$ & $<0.001$ & 1.175 & $1.112-1.242$ & $<0.001$ \\
\hline
\end{tabular}

$O R$ odds ratio, $C I$ confidence interval, $I C R B$ international classification of retinoblastoma, $E B R T$ external beam radiotherapy, TTT transpupillary thermotherapy. nonocular cancers were observed in two patients. One developed maxillary osteosarcoma and the other femur osteosarcoma at 180 and 72 months follow-up respectively after initial diagnosis.

\section{Extraocular and metastatic retinoblastoma}

Of 29 extraocular RB cases, 10 (34.5\%) had bilateral and 19 (65.5\%) had unilateral RB. Of 39 eyes with extraocular RB, 
14 eyes $(35.8 \%)$ had extraocular extension at presentation based on orbital biopsy and/or MRI features (thickened and infiltrated optic nerve and/or extrascleral mass together with intraocular RB) and 25 eyes $(64.1 \%)$ were found to have histopathologic evidence of extraocular extension (optic nerve cut end involvement and/or extrascleral extension) following enucleation.

Extraocular and metastatic RB were treated with Regimen A or Regimen B chemotherapy, EBRT to the brain, spine, orbit, and other relevant sites, and ASCR as necessary. Enucleation or exenteration was undertaken after chemotherapy/orbital EBRT in 5 of 14 eyes with evidence of extraocular extension at presentation. The mean followup was 39.7 (median: 15.5, range: 1-165) months. In total, 30 of $39(77 \%)$ extraocular RB eyes received enucleation or exenteration. Of 30 eyes, 14 were from bilateral RB patients and 16 were from unilateral RB patients. One patient with extraocular RB had trilateral $\mathrm{RB}$ and died from intracranial complications related to trilateral $\mathrm{RB}$.

In total, 12 of $29(41.4 \%)$ cases with extraocular RB were found to have metastasis to various sites and grouped as IRSS Group IVa (2 cases)/IVb (10 cases) while the rest $17(58.6 \%)$ were classified as Group II/III disease [3]. The metastatic sites were as follows: CNS (8 cases, 27.6\%), CNS and bone marrow (1 case, 3.4\%), CNS and lymph nodes (1 case, $3.4 \%$ ), and bone ( 2 cases, $6.9 \%$ ). Metastasis developed in 2 cases with only extrascleral extension but no cut-end optic nerve involvement. In total, 21 of 29 cases with extraocular RB were alive at the end of follow-up and survival was $72.4 \%$. Kaplan-Meier curve shows that survival was $79.2 \%$ at 1 year, $68.2 \%$ at 5 years, and $58.5 \%$ at 10 years for patients with extraocular RB (Fig. 1c). CNS metastasis had a bad outcome and 7 of 10 cases $(70.0 \%)$ with CNS metastasis died of the disease. One of 2 patients with bone metastasis also expired. The rest of the patients with CNS and distant metastasis were alive but continued to receive treatment.

\section{Trilateral retinoblastoma}

Three of $285(1.1 \%)$ patients had trilateral RB. Two of 3 (66.7\%) patients with trilateral RB had intraocular RB and 1 (33.3\%) had extraocular RB. In total, 2 of 3 patients, one patient with intraocular and one with extraocular RB, died from trilateral $\mathrm{RB}$.

\section{Overall globe conservation, metastasis, and death rates}

Of 351 intraocular RB eyes, 150 (42.7\%) underwent primary or secondary enucleation and $57.3 \%$ of the eyes could be salvaged. Of 276 eyes undergoing eye-conserving treatments, $201(72.8 \%)$ were preserved. Combining intraocular and extraocular RB cases together, 180 out of $390(46.2 \%)$ eyes underwent primary/secondary enucleation and exenteration. For eyes with bilateral RB, the enucleation/exenteration rate was $28.6 \%$ (63 of 210 eyes) versus $65.0 \%$ (117 of 180 eyes) for unilateral RB. Overall, globe salvage rate was $53.8 \%$ of all RB eyes.

Considering intraocular and extraocular RB together, 13 of 285 cases $(4.6 \%)$ had metastatic disease and $9(3.2 \%)$ expired. Two additional patients (one in intraocular RB and one in extraocular RB group) died from complications related to trilateral RB. Overall, 11 of 285 patients $(3.9 \%)$ died from metastatic and trilateral $\mathrm{RB}$ and the survival rate was $96.1 \%$.

Factors that were predictive of metastasis on univariate analysis were the presence of extraocular $\mathrm{RB}$ detected by imaging methods ( $p<0.001$, OR:193.286, 95\% CI: 44.021-848.663) and extrascleral/optic nerve cut end involvement at histopathological examination ( $p<0.001$, OR: 29.413, 95\% CI: 3.693-234.250).

Univariate risk factors predictive of death included the presence of vitreous seeds $(p=0.021$, risk ratio (RR): 11.133, 95\% CI: 1.447-85.632), presence of extraocular $\mathrm{RB}(p=0.021, \mathrm{RR}: 11.133,95 \%$ CI: 1.447-85.632), presence of extraocular RB detected by imaging methods ( $p<0.001$, RR: 50.280, 95\% CI: 16.236-155.713), and extrascleral/optic nerve cut end involvement at histopathological examination $(p=0.004, \mathrm{RR}: 20.603,95 \%$ CI: 2.574-164.928).

\section{Discussion}

$\mathrm{RB}$ is usually diagnosed at later ages in developing countries. In our study, the mean age of RB cases was 21.8 months similar to the results from the study of Mattosinho et al. from Brasil with a mean age of 21.7 months [5]. On the other hand, Gallie et al. reported that the mean age at diagnosis was 12 and 9 months for North America and Europe, respectively [6] and Shields et al. reported a mean age of 18 months from USA [7].

Although extraocular RB is rare in developed countries, it is not an unusual feature in the developing countries. Gao et al. reported that $91.3 \%$ of $\mathrm{RB}$ cases were intraocular and $8.7 \%$ were extraocular in a study from South Western China [8]. In our series, $10.2 \%$ of cases had extraocular RB at presentation reflecting a similar proportion. Chantada et al. reported that the frequency of extraocular $\mathrm{RB}$ in low middle income countries reaches $50 \%$ of newly diagnosed cases [9]. In other studies, extraocular RB comprised $18-40 \%$ of all $\mathrm{RB}$ cases at presentation in developing countries $[10,11]$.

The most commonly used treatment for RB in the past 25 years has been CRD. In our series, 276 eyes underwent eye- 
conserving treatments including CRD in the majority of cases and $201(72.8 \%)$ eyes could eventually be salvaged. Shields et al quoted globe salvage rates of $100 \%$ in group A, $93 \%$ in group B, $90 \%$ in group C, $47 \%$ in group D, and $25 \%$ in group $\mathrm{E}$ eyes at a median of 6.2 years follow-up following frontline CRD [4].

Unresponsive, recurrent or new tumours following CRD and focal treatments needed other treatments. In our series, $\mathrm{Ru}-106$ plaque radiotherapy was used in 18 (6.5\%) such eyes and $9(50.0 \%)$ were salvaged. Similarly, Murakami et al. reported an ocular salvage rate of 58.7\% after Ru-106 plaque radiotherapy in 90 eyes with RB [12]. In our study, EBRT was eventually needed in 48 (17.4\%) eyes and 33 $(68.7 \%)$ were rescued. Multivariable risk factors for treatment with EBRT after CRD included increasing age, advanced ICRB groups, and previous treatments including plaque radiotherapy and TTT. Shields et al. reported that the overall need for EBRT was $27.0 \%$ at 5 years [13]. Multivariable risk factors for EBRT included non-Caucasian vs Caucasian race, male versus female gender, and ReeseEllsworth group V vs group I-IV eyes in their study [13].

IAC was used in $25(9.1 \%)$ eyes in our series. IAC was employed as primary treatment in 3 eyes and all eyes were rescued. IAC was used as secondary salvage treatment after failed CRD and focal treatments in 22 eyes and 10 of 22 $(45.4 \%)$ were rescued. Significantly better results have been reported using IAC at other centres and Francis et al. reported that none of the 64 naïve bilateral $\mathrm{RB}$ eyes receiving IAC were enucleated [14]. Shields et al. quoted that $74 \%$ of unilateral RB eyes could be saved after primary IAC [15].

The most challenging aspect of RB treatment is control of subretinal and especially vitreous seeds. IAC has largely been ineffective in treating vitreous seeds [16]. For more than 75 years, EBRT was the only treatment method for vitreous seeding. In eyes with refractory vitreous seeds, intravitreal delivery of chemotherapeutic agents has emerged as a promising choice of treatment. Currently, melphalan and topotecan are the most extensively used intravitreal drugs in the treatment of vitreous seeds in $\mathrm{RB}$ [17]. Munier et al. noted regression of vitreous seeds in $87 \%$ of eyes treated with intravitreal melphalan [17]. A study by Abramson et al. showed that IVC had an expanded spectrum and was successful at achieving regression of both retinal tumours, subretinal seeds, and subretinal fluid in addition to vitreous seeds [18]. In our series, IVC was used as secondary treatment in $11(4.0 \%)$ eyes with refractory vitreous seeds following other treatments and 8 of 11 $(72.7 \%)$ eyes were salvaged.

In our study, 59 of 276 (21.4\%) eyes with intraocular RB undergoing eye conserving treatments demonstrated new tumours, and 71 of $276(25.7 \%)$ eyes developed recurrent tumours. The mean times to the development of new and recurrent tumours were 9.7 and 11.4 months after initiation of CRD, respectively. These findings emphasize the importance of long term follow-up in intraocular RB after CRD and focal treatments.

A total of 150 eyes with intraocular RB underwent primary and secondary enucleation in our series. For eyes with unilateral $\mathrm{RB}$, the enucleation rate was $62.7 \%$ (101 of 161 eyes) compared with $27.2 \%$ (49 of 190 eyes) for bilateral RB. Bonanomi et al. found that the enucleation rate was $93.3 \%$ in eyes with unilateral RB versus 50\% in bilateral RB [19]. Gao et al. similarly reported that the overall enucleation rate $90 \%$ for unilateral $\mathrm{RB}$ and $46 \%$ for bilateral $\mathrm{RB}$ [8]. It appears that unilateral $\mathrm{RB}$ behaves differently compared to bilateral RB and enucleation rate for unilateral disease is higher worldwide. Combining intraocular and extraocular RB, the overall enucleation/exenteration rate was $46.2 \%$ and globe salvage was achieved in $53.8 \%$ of the eyes in our series. The fact that $53.3 \%$ of the eyes were group D and E at presentation and $10.0 \%$ of all eyes had extraocular RB may have contributed to the lower ocular salvage rate in our conditions.

Multivariable risk factors for enucleation in our series included larger tumour, presence of vitreous seeds, no prior EBRT, and fewer focal treatments. Shields et al. reported that multivariable factors associated with enucleation were age at diagnosis of $\mathrm{RB}>12$ months, single tumour, and tumour distance to foveola $\leq 2 \mathrm{~mm}$ [13].

Metastatic RB still remains as the major cause of mortality from RB worldwide. The frequency of metastatic RB was quoted at 9 and $11 \%$ in two reports from developing countries $[9,20]$. In our study, the rate of systemic metastasis from RB was $4.6 \%$. Tumour at the cut end of the optic nerve is the most important risk factor for CNS metastasis in RB [21]. Retrolaminar optic nerve involvement especially when it occurs with $>3 \mathrm{~mm}$ choroidal invasion was also found to be a risk factor for CNS metastasis [21]. In our study, one case with histopathologically determined retrolaminar optic nerve involvement developed CNS metastasis. Central nervous system involvement can also be seen in cases who have extraocular spread without optic nerve involvement [21]. In 2 patients in our series, CNS involvement developed after extrascleral spread without optic nerve involvement. Central nervous system metastasis seems to have a fatal outcome despite intensive chemotherapy and EBRT in many cases. However, other metastatic sites such as lymph nodes, bone, and bone marrow can be cured with intensive chemotherapy and EBRT with variable success. Therefore, early diagnosis and treatment of RB before it extends outside the eye through the sclera and optic nerve is important. Our study showed that the parameters that were predictive of metastasis on a univariate level were extraocular $\mathrm{RB}$ detected by imaging methods and extrascleral/optic nerve cut end involvement detected at histopathological examination. In our study, the risk of metastasis was approximately $<1 \%$ for intraocular $\mathrm{RB}$ and $41 \%$ for extraocular RB. 
In developed countries, the survival rate of children with RB has improved over the years. However, in lessdeveloped countries, the survival rate is lower, with mortality rates of nearly $40 \%$ in Asia and $70 \%$ in Africa [1]. The high mortality rate in less-developed countries is mainly related to the lack of access to adequate health care services, poor socioeconomic conditions, and delayed diagnosis all resulting in advanced disease at presentation. In an analysis of gross national income of the country versus RB mortality, Chantada et al. reported that survival from RB is $30 \%$ in low income countries, $60 \%$ in lower middle income countries, $75 \%$ in upper middle income countries, and $95 \%$ in high income countries [9]. In our series, the overall survival rate was $96.1 \%$ which approximates the level in high income developed countries despite the fact that Turkey was listed as an upper middle income country as of 2018 (http:// data.worldbank.org/country/turkey).

The limitations of our study include its retrospective nature and possible lack of standardized treatment in similar patients because of patient considerations and family preferences. The treatment of RB has undergone significant changes from enucleation and EBRT to CRD over the past 25 years. As a further advancement, the addition of IAC and IVC to the treatment armamentarium decreased the need for EBRT, plaque radiotherapy, and enucleation during the last 10 years. In our study, $\sim 73 \%$ of the eyes with intraocular RB undergoing various eye-conserving methods were preserved, most of them undergoing intravenous chemotherapy and/or focal treatments initially. However, our audit report over the recent 20 years period from a major referral center in a developing middle income country show that $\sim 53 \%$ of intraocular RB eyes were group D and $\mathrm{E}$ at presentation and that globe salvage was possible in only $54 \%$ of all intraocular and extraocular RBs.

\section{Summary}

\section{What was known before}

- Recent papers on retinoblastoma (RB) concentrate on outcomes after newer treatments including intraarterial and intravitreal chemotherapy in selected intraocular RB patients but do not evaluate those in whom these treatments may not be applicable. Further, long term treatment outcomes for the whole cohort of intraocular and extraocular RB patients is lacking in many middle income developing countries.

\section{What this study adds}

- This study provides relatively long term treatment outcomes for both intraocular and extraocular RB patients over 20 years at one of the largest and most frequently referred tertiary care centers in Turkey. For intraocular RB, it assesses the risks for eventual treatment with external beam radiotherapy and enucleation in the intravenous chemotherapy era, with intraarterial/intravitreal chemotherapy added to the treatment list in the last 10 years.

- Our study also predicts the risk of death in extraocular RB patients, which is not an uncommon situation in many countries outside North America and Europe. It provides real life data from a middle income developing country.

Acknowledgements There are no sources of support including sponsorship (e.g. university, charity, commercial organization) and sources of material (e.g. novel drugs) not available commercially.

\section{Compliance with ethical standards}

Conflict of interest The authors declare that they have no conflict of interest.

Publisher's note Springer Nature remains neutral with regard to jurisdictional claims in published maps and institutional affiliations.

\section{References}

1. Kivelä T. The epidemiological challenge of the most frequent eye cancer: retinoblastoma, an issue of birth and death. Br J Ophthalmol. 2009;93:1129-31.

2. Shields CL, Shields JA. Diagnosis and management of retinoblastoma. Cancer Control. 2004;11:317-27.

3. Chantada G, Doz F, Antoneli CB, Grundy R, Clare Stannard FF, Dunkel IJ, et al. A proposal for an international retinoblastoma staging system. Pediatr Blood Cancer 2006;47:801-5.

4. Shields CL, Mashayekhi A, Au AK, Czyz C, Leahey A, Meadows AT, et al. The international classification of retinoblastoma predicts chemoreduction success. Ophthalmology. 2006;113:2276-80.

5. Mattosinho CCDS, Grigorovski N, Lucena E, Ferman S, Soaresde Moura ATM, Portes AF. Prediagnostic intervals in retinoblastoma: experience at a noncology center in Brazil. J Glob Oncol. 2016;3:323-30.

6. Gallie BL, Zhao J, Vandezande K, White A, Chan HS. Global issues and opportunities for optimized retinoblastoma care. Pediatr Blood Cancer. 2007;49:1083-90.

7. Shields JA, Shields CL. Retinoblastoma. In: Shields JA, Shields CL (eds). Intraocular Tumors. An Atlas and Textbook (2nd ed). Lippincott Williams Wilkins: Philadelphia, PA; 2008: 293-365.

8. Gao J, Zeng J, Guo B, He W, Chen J, Lu F, et al. Clinical presentation and treatment outcome of retinoblastoma in children of South Western China. Medicine. 2016;95:e5204.

9. Chantada GL, Qaddoumi I, Canturk S, Khetan V, Ma Z, Kimani $\mathrm{K}$, et al. Strategies to manage retinoblastoma in developing countries. Pediatr Blood Cancer. 2011;56:341-8.

10. Ali MJ, Honavar SG, Reddy VA. Orbital retinoblastoma: present status and future challenges-A review. Saudi J Ophthalmol. 2011;25:159-67.

11. Badhu B, Sah SP, Thakur SK, Dulal S, Kumar S, Sood A, et al. Clinical presentation of retinoblastoma in Eastern Nepal. Clin Exp Ophthalmol. 2005;33:386-9. 
12. Murakami N, Suzuki S, Ito Y, Yoshimura R, Inaba K, Kuroda Y, et al. 106 ruthenium plaque therapy (RPT) for retinoblastoma. Int J Radiat Oncol Biol Phys. 2012;84:59-65.

13. Shields CL, Honavar SG, Meadows AT, Shields JA, Demirci H, Singh A, et al. Chemoreduction plus focal therapy for retinoblastoma: factors predictive of need for treatment with external beam radiotherapy or enucleation. Am J Ophthalmol. 2002; 133:657-64.

14. Francis JH, Roosipu N, Levin AM, Brodie SE, Dunkel IJ, Gobin YP, et al. Current treatment of bilateral retinoblastoma: the impact of intraarterial and intravitreous chemotherapy. Neoplasia. 2018;20:757-63.

15. Shields CL, Jorge R, Say EA, Magrath G, Alset A, Caywood E, et al. Unilateral retinoblastoma managed with intravenous chemotherapy versus intra-arterial chemotherapy. Outcomes based on the international classification of retinoblastoma. Asia Pac J Ophthalmol. 2016;5:97-103.

16. Shields CL, Bianciotto CG, Jabbour P, Ramasubramanian A, Lally SE, Griffin GC, et al. Intra-arterial chemotherapy for retinoblastoma: report no. 1, control of retinal tumors, subretinal seeds, and vitreous seeds. Arch Ophthalmol. 2011;129:1399-406.

17. Munier FL, Gaillard MC, Balmer A, Soliman S, Podilsky G, Moulin AP, et al. Intravitreal chemotherapy for vitreous disease in retinoblastoma revisited: from prohibition to conditional indications. Br J Ophthalmol. 2012;96:1078-83.

18. Abramson DH, Ji X, Francis CH, Catalanotti F, Catalanotti F, Brodie SE, et al. Intravitreal chemotherapy in retinoblastoma: expanded use beyond intravitreal seeds. $\mathrm{Br} \mathrm{J}$ Ophthalmol. 2019;103:488-93.

19. Bonanomi MT, Almeida M, Cristofani LM, Odone Filho V. Retinoblastoma: a three-year-study at a Brazilian medical school hospital. Clinics. 2009;64:427-34.

20. Leal-Leal C, Flores-Rojo M, Medina-Sansón A, Cerecedo-Díaz F, Sánchez-Félix S, González-Ramella $\mathrm{O}$, et al. A multicentre report from the Mexican Retinoblastoma Group. Br J Ophthalmol. 2004;88:1074-7.

21. Finger PT, Harbour JW, Karcioglu ZA. Risk factors for metastasis in retinoblastoma. Surv Ophthalmol. 2002;47:1-16. 